\title{
Article
}

\section{On demand manufacturing of patient- specific liquid capsules via co-ordinated 3D printing and liquid dispensing}

Okwuosa, Tochukwu Chijioke, Soares, Cindy, Golwitzer, Verena, Habashy, Rober, Timmins, Peter and Albed Alhnan, Mohamed Available at http://clok.uclan.ac.uk/22021/

Okwuosa, Tochukwu Chijioke, Soares, Cindy, Golwitzer, Verena, Habashy, Rober, Timmins, Peter and Albed Alhnan, Mohamed (2018) On demand manufacturing of patient-specific liquid capsules via co-ordinated 3D printing and liquid dispensing. European Journal of Pharmaceutical Sciences, 118 . pp. 134-143. ISSN 0928-0987

It is advisable to refer to the publisher's version if you intend to cite from the work. http://dx.doi.org/10.1016/j.ejps.2018.03.010

For more information about UCLan's research in this area go to http://www.uclan.ac.uk/researchgroups/ and search for <name of research Group>.

For information about Research generally at UCLan please go to http://www.uclan.ac.uk/research/

All outputs in CLoK are protected by Intellectual Property Rights law, including Copyright law. Copyright, IPR and Moral Rights for the works on this site are retained by the individual authors and/or other copyright owners. Terms and conditions for use of this material are defined in the policies page. 


\section{On Demand Manufacturing of Patient-Specific Liquid Capsules via co-ordinated 3D Printing and Liquid Dispensing}

Tochukwu C Okwuosa ${ }^{1}$, Cindy Soares ${ }^{1}$, Verena Gollwitzer ${ }^{1,2}$, Rober Habashy ${ }^{1}$, Peter Timmins $^{3}$, Mohamed A Alhnan ${ }^{1^{*}}$

${ }^{1}$ School of Pharmacy and Biomedical Sciences, University of Central Lancashire, Preston, Lancashire, UK.

2 Department of Chemistry and Pharmacy, Friedrich-Alexander University ErlangenNürnberg, Erlangen, Germany.

${ }^{3}$ Department of Pharmacy, University of Huddersfield, Huddersfield, UK.

*Corresponding author: MAlbedAlhnan@uclan.ac.uk 


\begin{abstract}
A method for the production of liquid capsules with the potential of modifying drug dose and release is presented. For the first time, the co-ordinated use of fused deposition modelling (FDM), 3D printing and liquid dispensing to fabricate individualised dosage form on demand in a fully automated fashion has been demonstrated. Polymethacrylate shells (Eudragit EPO and $R L$ ) for immediate and extended release were fabricated using FDM 3D printing and simultaneously filled using a computer-controlled liquid dispenser loaded with model drug solution (theophylline) or suspension (dipyridamole). The impact of printing modes: simultaneous shell printing and filling (single-phase) or sequential 3D printing of shell bottom, filling and shell cap (multi-phase), nozzle size, syringe volume, and shell structure has been reported. The use of shell thickness of $1.6 \mathrm{~mm}$, and concentric architecture allowed successful containment of liquid core whilst maintaining the release properties of the 3D printed liquid capsule. The linear relationship between the theoretical and the actual volumes from the dispenser reflected its potential for accurate dosing $\left(\mathrm{R}^{2}=0.9985\right)$. Modifying the shell thickness of Eudragit RL capsule allowed a controlled extended drug release without the need for formulation change. Owing to its low cost and versatility, this approach can be adapted to wide spectrum of liquid formulations such as small and large molecule solutions and obviate the need for compatibility with the high temperature of FDM 3D printing process. In a clinical setting, health care staff will be able to instantly manufacture in small volumes liquid capsules with individualised dose contents and release pattern in response to specific patient's needs.
\end{abstract}

Key words: Rapid prototyping; precision medicine; capsulation, personalised; patientspecific; three-dimensional printing; additive manufacturing 


\section{Introduction}

Personalised dosing is an upcoming and promising approach in drug therapy that ensures doses are tailored to an individual patient's needs and preferences (McDougall et al., 2016). This can reduce the incidence of side effects and risk of overdose as pharmacodynamic and pharmacokinetic factors are able to be considered along with the age and weight of the patient (Al-Metwali and Mulla, 2017). Over the years, individualising liquid oral dosage forms e.g. solutions and suspensions has been carried out by a variety of simple dosing aids e.g. calibrated spoons, droppers or syringes. Although these methods provided a low-cost solution, they were however associated with human errors during dosing (Ryu and Lee, 2012; Sobhani et al., 2008).

Liquid capsules have been used to enhance the absorption of poorly soluble drugs (Cole et al., 2008; Deepthi and Murthy, 2015; Hussein et al., 2012). The use of hard gelatine capsules can offer a significant advantage over soft gel capsules for encapsulation of liquids and semisolids. There is a reduced incidence of drug migration (Armstrong et al., 1984) and an improvement in product stability by lowering moisture and oxygen permeability, rendering it more suitable for sensitive active molecules (Hom et al., 1975; Lucas et al., 1987). Hard shell capsules also provide better taste and odour masking, and hence can improve patient's compliance. The wide range of application of capsules in medical treatment are already well established; however, dose personalisation using liquid capsules is yet to be investigated.

The pharmaceutical applications of 3D printers in pharmaceutical production have demonstrated great potential as an alternative manufacturing technique for personalising dosage forms at a peripheral level. 3D printing has been used in the manufacturing of immediate (Okwuosa et al., 2016; Pietrzak et al., 2015; Sadia et al., 2016), extended (Chai et al., 2017; Clark et al., 2017; Goyanes et al., 2015; Kyobula et al., 2017; Park, 2015; Skowyra et al., 2015), as well as enteric release (Goyanes et al., 2017; Okwuosa et al., 2017) dosage forms using pharmaceutical grade polymers. In FDM 3D printing, filaments are heated above the glass transition temperature $(\mathrm{Tg})$ of the matrix polymer, passed down a nozzle by gears and deposited in a layer-by-layer fashion to fabricate an object without the need for postprinting processing (Alhnan et al., 2016).

In a previous attempt, FDM 3D printing was used in the fabrication of capsule by interrupting the printing of the shell and filling in the core manually before completing the shell (Markl et al., 2017). In another example, the capsule cap and bottom were 3D printed separately and then filled manually with powder (Melocchi et al., 2015). To the authors' knowledge, there have been no previous reports around a fully integrated, automated 3D printing of liquid capsules. This could be a reflection of major challenges associated with attempts to achieve this goal. 
Unlike manufacturing capsules by casting, the additive manufacturing of capsules imposes a major difficulty of sealing the spaces between the printed layers and maintaining the barrier function of the shell. In fact, a fully automated 3D printing of liquid capsules requires the coordination of a liquid dispenser with the shell manufacturing. The design of the shells, its additive manufacturing and liquid filling should be carefully organised to ensure that the resultant shell structure are compatible with the liquid core.

In this research, we demonstrate the first example of a fully automated additive manufacturing process for a liquid capsule with the capability to control the dispensed dose. A dual FDM 3D printer has been modified to include a syringe based liquid dispenser. This was used to fabricate a capsule shell through FDM 3D printing and instantaneously dispense either a suspension or a solution formulation of model drugs. The dosing accuracy of the liquid dispenser was optimised and used in the manufacturing of immediate or extended release liquid capsules employing two methacrylate polymers. Eudragit EPO is an amino alkyl methacrylate copolymer, which is soluble at $\mathrm{pH} \leq 5$. It has been used as a taste-masking agent (Taki et al., 2017) and provides a glossy and slippery easy-to-swallow finishing. Its use is well established for pharmaceutical purposes as a solubility enhancer (Higashi et al., 2016; Saal et al., 2017, 2018), stability enhancer (Ochi et al., 2016) and in the manufacturing of immediate release dosage forms (Qiao et al., 2010; Sadia et al., 2016; Yang et al., 2015). Its compatibility with hot melt extrusion has also been previously demonstrated (Ashour et al., 2016; Li et al., 2016a; Li et al., 2016b). On the other hand, Eudragit $R L$ is an insoluble and permeable polymer, which has been used for time-controlled drug release (Dave et al., 2015; Elzayat et al., 2017; Ozguney et al., 2007).

\section{Materials and methods}

\subsection{Materials}

Theophylline (>99\%, anhydrous) was purchased from Acros Organics (UK). Triethyl citrate (TEC), Tween 80 , citric acid and dipyridamole ( $\geq 98 \%$ ) were purchased from Sigma-Aldrich (UK). Talc was purchased from Fluka Analytical (UK). Methocel E4 was donated by Colorcon limited (UK). Amino alkyl methacrylate copolymers (Eudragit EPO and Eudragit RL) were donated by Evonik Industries (Darmstadt, Germany).

\subsection{Preparation of shell filament}

For the preparation of the capsule shell, drug-free Eudragit EPO or RL filaments were produced by a HAAKE MiniCTW hot melt compounder (Thermo Scientific, Karlsruhe, Germany). An optimised ratio of a powder mixture constituting of the polymer, plasticizer (TEC) and filler (talc) was gradually added to the HME and allowed to mix for 5 min at 80 
rpm to allow homogenous distribution of the molten mass. Afterwards, the filament was extruded at $20 \mathrm{rpm}$. The processing parameters for the hot melt extrusion are shown in Table 1.

\subsection{Thermal analysis}

For differential scanning calorimetry (DSC) analysis of Eudragit EPO and RL, a differential scanning calorimeter (DSC) Q2000 (TA Instruments, Elstree, Hertfordshire, UK) with a heating rate of $10^{\circ} \mathrm{C} / \mathrm{min}$ was employed. Each sample was subjected to a heat-cool-heat scan in order to measure and exclude the effect of moisture contents on filament plasticity. A standard scan was carried out from -70 to $200^{\circ} \mathrm{C}$ or to $150{ }^{\circ} \mathrm{C}$ for Eudragit EPO and RL respectively. Analysis was carried out under a purge of nitrogen $(50 \mathrm{~mL} / \mathrm{min})$. The data was analysed using a TA 2000 analysis software. Each sample (approximately $5 \mathrm{mg}$ ) was accurately weighed and placed in a $40 \mu \mathrm{L}$ aluminium pan and covered with pin-holed lid (TA Instruments, Elstree, Hertfordshire, UK). All measurements were carried out in triplicate.

For TGA analysis, 3D-printed capsule shells, raw materials as well as extruded filaments were investigated using a TGA Q5000 (TA Instruments, Hertfordshire, UK) as described in a previous study (Okwuosa et al., 2016).

\subsection{X-ray powder diffraction}

A powder X-ray diffractometer, D2 Phaser with Lynxeye (Bruker, Germany) was used to assess the physical properties of the shell filaments using parameters as reported earlier (Okwuosa et al., 2016).

\subsection{Scanning Electron Microscopy (SEM)}

A JOEL JCM-6000PLUS benchtop SEM microscope (Joel LTD, Tokyo, Japan) was used to examine the surface morphology of the printed shell-core structures. Images of the capsules were also taken using a Canon EOS-1D Mark IV (Canon Ltd, Japan).

\subsection{Preparation of the liquid core}

Two model liquid cores (aqueous active suspension or solution) were prepared for use in the syringe based liquid dispenser:

a. Dipyridamole suspension was initially prepared by subjecting aqueous dipyridamole suspension ( $1.5 \mathrm{~g} / 30 \mathrm{~mL}$ ) to size reduction via application to T8.01 Ultra Turrax Homogeniser (IKA, Germany) at 25,000 rpm. This was carried out for $1 \mathrm{~h}$ at $15 \mathrm{~min}$ interval with $5 \mathrm{~min}$ cooling time between the intervals. Methocel E4 was added to the suspension to reach a polymer concentration $0.5 \% \mathrm{w} / \mathrm{v}$ before probe sonicating using Sonics Vira cell (USA) at 15 min interval 
in an ice bath for additional $4 \mathrm{~h}$ using an amplitude of $70 \%$. The final suspension was diluted with Methocel E4 $(0.5 \% \mathrm{w} / \mathrm{v}$ in water) to achieve a drug concentration of $1.5 \% \mathrm{w} / \mathrm{v}$. The size distribution of dipyridamole particles in the suspension was confirmed by a Mastersizer 2000 laser diffraction particle size analyser (Malvern Instruments, UK).

b. Theophylline solution was prepared by adding $15 \mathrm{~g}$ of citric acid and $1.5 \mathrm{~g}$ of Tween 80 to a $4 \% \mathrm{w} / \mathrm{v}$ theophylline aqueous suspension $(50 \mathrm{~mL})$. This was heated to $65^{\circ} \mathrm{C}$ and stirred until a complete solution is formed. Methocel E4 was then added to achieve a cellulosic solution concentration of $0.25 \% \mathrm{w} / \mathrm{v}$ before cooling in an ice bath.

\subsection{Modification of dual FDM 3D printer}

In order to devise a fully automated manufacturing of the liquid capsule, a Makerbot Replicator Experimental 2X dual FDM 3D printer (MakerBot Industries, New York, USA) was modified. The printer has two FDM nozzle heads. The right extruder/head of the dual 3D printer was replaced by a syringe-based liquid dispenser as shown in Supplementary Data, Fig 1S. The design for the dispenser was obtained from an open source design (Thingiverse, 2017) and the different parts were produced by 3D printing using an M2 Makergear FDM 3D printer and ABS filaments (MakerGear LLC, Ohio, USA). The dispenser head was assembled and equipped with either a 2 or $10 \mathrm{~mL}$ syringe. A Nema17 1.5A 4-lead stepper motor (MakerBot Industries, New York, USA) was connected to the motherboard using the default housing connectors.

\subsection{Liquid Capsule design and printing}

The shells of the capsules were designed as a $1.6 \mathrm{~mm}$ thick capsule shell with a capsuleshaped cavity and different dimensions (Table 2). Regardless of the digital design of the core, the core will be dispensed as a liquid and will fill the cavity of the capsule shell. A simple geometry (cube) was chosen as the digital design for the core to simplify the calculations of core's volume and to confine the movement of the liquid dispenser head within the cavity space. This proved to be a more suitable approach than using capsule-shaped digital design for the core. The contents of the core were manipulated by modifying the dimensions of the digital design of the core as specified in section 2.9 and 2.10 .

For the fabrication of liquid capsule, two different printing modes were employed (Fig. 2):

a. Single-phase printing: Within the Makerbot Desktop software version 3.10.0.1364 (MakerBot Industries, New York, USA), the core was placed in the centre of the cavity of its 
corresponding shell and was printed by the interchanging printing of the shell filament and core liquid.

b. Multi-phase printing: A simplified 3D software version 3.1.1 (Simplify 3D LLC, Ohio, USA) was used in this printing mode. The shell was designed to comprise a complementary bottom and a cap. This liquid capsule printing was carried out in 3 phases: i) printing of the shell bottom, ii) filling of liquid core, and iii) sealing of the shell in a separate 3D printing stage.

The liquid capsules for both modes were printed with cube dimensions corresponding to 80 , 160,240 or $320 \mu \mathrm{L}$ (Table 2). The settings of the software were modified, and the shells were printed using the 3D printing parameters as shown in Table 1. The resolution was set at medium (200 $\mu \mathrm{m}$ layer thickness), the infill was $100 \%$ and the internal and external infill pattern were set at grid and concentric respectively. The rest of the settings were left as default. As priming was not necessary for liquids, the script of the software was also modified to omit the priming step of the liquid dispenser.

\subsection{Validation of the liquid dispenser using different nozzle sizes}

The impact of different nozzle sizes on liquid volumes from the liquid dispenser was investigated using digital design of core with cubes of edge dimensions: $2.15,2.71,3.42,4.31$, $4.93,5.43,5.85$, and $6.21 \mathrm{~mm}$ as the printed object. These dimensions corresponded to theoretical volumes of $10,20,40,80,120,160,200$ and $240 \mu \mathrm{L}$. A $10 \mathrm{~mL}$ syringe was then used for drug dispensing using $0.25,0.41$ or $0.84 \mathrm{~mm}$ nozzle sizes. The dispensed liquid was collected, weighed and the volume determined based on the density of the liquid at the operating temperature. The relationship between the theoretical volumes and the actual dispensed liquid volumes were determined. The effect of different 3D printing modes (singlephase or multi-phase) on the accuracy of the dispensed volume was also investigated.

\subsection{Optimising the dosing accuracy of the liquid dispenser using different syringe sizes}

The dosing accuracy of the liquid dispenser was investigated using dispensed liquids corresponding to digital design of the core (cube volume of $80,160,240$ or $320 \mu \mathrm{L}$ ). The nozzle size was $0.41 \mathrm{~mm}$ and the effect of syringe sizes (2 or $10 \mathrm{~mL}$ ) (Nipro Luer Lock) was investigated $(n=4)$. The dispensed liquid was collected into a tared $5 \mathrm{~mL}$ polystyrene diamondshaped weighing-boat (Fisher scientific, UK) and weighed. The estimated dispensed volumes were calculated based on the density of the dispensed volume. The estimated doses were calculated based on the concentration of the suspension and the estimated dispensed volume. The actual dispensed dose was determined using HPLC methods shown in section 2.11. The 
dosing efficiency was used to compared the estimated doses versus actual doses and was calculated using Equation 1.

Efficiency $(\%)=($ estimated dose/actual dose $) \times 100$

Equation 1

The relationship between the estimated and actual and doses was determined using both printing modes. Due to higher accuracy of $2 \mathrm{~mL}$ syringe and the superior quality products produced via multi-phase mode, they were considered as a default and used for the 3D printing of all capsules reported for dissolution tests.

\subsection{Determination of drug contents}

Liquid capsules and their contents were placed in a $500 \mathrm{~mL} \mathrm{1:1} \mathrm{acetonitrile} \mathrm{and} \mathrm{water} \mathrm{in} \mathrm{a}$ volumetric flask and then sonicated for $2 \mathrm{~h}$. The solution was filtered through $0.22 \mu \mathrm{m}$ MillexGP syringe filters (Merck Millipore, USA) and prepared for HPLC analysis. Dipyridamole and theophylline contents in relevant samples were assessed using an Agilent UV-HPLC 1260 series (Agilent Technologies Inc., Germany) equipped with XTerra RP 18 column (150 × $4.6 \mathrm{~mm}, 5 \mu \mathrm{m}$ particle size) (Waters, Ireland) as previously reported (Okwuosa et al., 2016).

\subsection{In vitro Dissolution Test}

In vitro drug release studies for all liquid capsules used in this study were carried out in triplicate in $900 \mathrm{~mL}$ of $0.1 \mathrm{~N} \mathrm{HCl}$ at $37 \pm 0.5{ }^{\circ} \mathrm{C}$ in USP II apparatus (AT7, Sotax, city, Switzerland) with a paddle speed of $50 \mathrm{rpm}$. The capsules were placed in sinkers to ensure immersion in dissolution medium.

a. For the Eudragit E-dipyridamole liquid capsule release studies, four $\mathrm{mL}$ aliquots of release medium were manually collected using $5 \mathrm{~mL}$ Leur-Lock syringes at $0,5,10,15,20,25,30$, 40 and 60 min time intervals and filtered through a Millex-HA $0.45-\mu \mathrm{m}$ filter. Each aliquot withdrawn was replaced with $4 \mathrm{~mL}$ of $0.1 \mathrm{M} \mathrm{HCl}$. Analysis of dipyridamole content of samples was undertaken using HPLC methods reported in section 2.11.

b. Eudragit E-theophylline liquid capsule release studies were conducted using an in-line UV/VIS spectrophotometer (PG Instruments Limited, UK). The amount of released theophylline was determined at $5 \mathrm{~min}$ intervals at a wavelength of $272 \mathrm{~nm}$ and path length of $1 \mathrm{~mm}$. Data was analysed using IDISis software (Automated Lab, 2012).

c. For Eudragit RL-Theophylline extended release liquid capsules, the test was carried out using $750 \mathrm{~mL}$ of a stimulated gastric fluid $(0.1 \mathrm{M} \mathrm{HCl}, \mathrm{pH} 1.2)$ for $2 \mathrm{~h}$ followed by $12 \mathrm{~h}$ exposure to $\mathrm{pH} 6.8$ phosphate buffer. 


\subsection{Statistical Analysis}

One-way ANOVA was employed using SPSS Software (22.0.0.2) to analyse the results. Differences in results above probability level $(p>0.05)$ was considered not significant whilst differences $(p<0.05)$ were considered significant. 


\section{Results and Discussion}

The co-ordination of a FDM 3D printer with a liquid dispenser enabled the manufacturing of liquid capsules in a fully automated process (Figure 1). The proposed configuration allowed the synchronisation of two processes: i) capsule shell fabrication, and ii) capsule filling whilst maintaining control over both through 3D printing software. To orchestrate these processes, two modes of capsule shell printing and filling have been adapted. The single-phase 3D printing, which involves the simultaneous construction of the shell and dispensing of the liquid core into its cavity (Figure 2A, Supplementary Data Video S1). However, this mode implicates a constant switching between the two printing heads after building each layer, leading to frequent disruptions of the shell 3D printing process. It is possible that core liquids can interfere with the layer-by-layer deposition of filaments during shell manufacturing by the deposition of liquid on the growing layers, hence preventing the casted layers from fusing and in effect compromising the barrier function of the shell walls. This was avoided when a multiphase printing mode was adopted. In multi-phase printing, the printing of $75 \%$ of the bottom side of the shell is first completed, followed by the core liquid filling before sealing the shell in another separate 3D printing stage (Figure 2B, Supplementary Data Video S2).

The design and the printing patterns of the shell were essential to successfully print liquid capsules. The capsule shell was hollow in the middle with a $1.6 \mathrm{~mm}$ thick wall to be able to accommodate the liquid core (Figure 2). When thinner wall thicknesses were initially assessed, it led to leakage of the liquid core during or following the printing process and were deemed insufficient to control liquid contents within the shell (unpublished data).

The thermogravimetric analysis of the shell demonstrated that the filaments were not subjected to a significant weight loss at the 3D printing temperatures (Fig. 3 A1 and A2). The DSC analysis of the filaments indicated that the addition of TEC to the methacrylate polymeric matrix in filament manufacturing effectively plasticised the polymers and lowered their Tg from 43.7 to $18.9^{\circ} \mathrm{C}$ and from 74.7 to $47.9^{\circ} \mathrm{C}$ for Eudragit EPO and RL respectively (Fig. 3 B1 and B2). The resultant filaments were thus compatible with FDM 3D printer head. The addition of the structure former, talc, was essential to permit the 3D printing by regulating the filament diameter, retaining the filament integrity following going through nozzles and allowing rapid solidification after extrusion from the nozzle (Okwuosa et al., 2016). With these adaptations, it was possible to carry out FDM 3D printing at 135 and $170{ }^{\circ} \mathrm{C}$ for Eudragit EPO and RL respectively.

The XRD patterns for the filaments and 3D printed capsule shell based on both Eudragit EPO and $R \mathrm{~L}$ demonstrated the presence of peaks at $\left(2^{\theta}\right)=9.5,19.0,28.6,36.5$ and $48.9^{\circ}$ (Fig.4). These peaks could be attributed to the talc used in the formulation, and confirmed that talc 
remained in a crystalline form. Previous research also indicated that in filled polymeric systems, the addition of a non-melting component to methacrylic polymer enhanced the viscoelastic behaviour of the melt in a monotonic fashion and decreased the critical strain amplitude for strain thinning (Sadia et al., 2016).

In conventional capsule filling, most gelatine capsules are designed at thicknesses of usually $\leq 0.240 \mathrm{~mm}$, which could differ depending on the size and the manufacturer of the capsules (Capsugel, 2012; Limited, 2014). The body and the cap of gelatine capsules are manufactured separately, typically by casting before being filled and sealed. In this research, the shell and the core of a 3D printed liquid capsule were created simultaneously by stacking polymeric layers and filling the printed cavity with liquid. Therefore, the barrier function of the 3D printed capsule shell was maintained by increasing shell thickness to $1.6 \mathrm{~mm}$ and applying a $100 \%$ infill pattern. Moreover, the pattern of shell printing also seemed to influence the integrity of the shell. While rectilinear pattern resulted in gaps of approximately $100 \mu \mathrm{m}$ (Figs. 5A1 and 5A2), concentric capsule filling provided tighter alignment of the fused filament and resulted in more stable shell (Figs. 5B1 and 5B2).

The printing mode appeared to influence the architecture and the finishing of the capsule. In multi-phase printing mode, the wall of the shell appeared tighter in comparison to single-phase printing (Figs. 6A1 and 6B1). In single-phase mode, following the deposition of each layer, the FDM 3D printer's nozzle moves away from the shell to allow the liquid dispenser to fill the capsule. To add the next layer, the nozzle head returns to the same $x-y$ spot at a higher level (200 $\mu \mathrm{m}$, thickness of the one layer). However, the lag in the stoppage and commencement of material flow from the printer's nozzle during heads movement and alternation results in the formation of a gap at each layer starting point (Fig. 6A2). By employing multi-phase 3D printing, it was possible to minimize imperfection (Fig. 6B2) as the 3D printing of the shell is only interrupted once. It is expected that the precision of the printing will improve with the development of highly precise 3D printers (Gross et al., 2014).

These different modes of 3D printing appear to also affect the volume of dispensed liquid ( $p<$ 0.05) (Fig. 7A and 7B). The lower dispensed volume from the single-phase mode compared to multi-phase printing could be attributed to the continuous stoppage and retraction of the liquid dispenser at each layer of printing (as a part of switching between FDM printing nozzle and liquid dispenser). This creates a gap between dispensed volumes leading to a lower overall volume. Therefore, the multi-phase mode was employed as a default printing method.

As accurate dose dispensing is of paramount importance for personalised dosing, it was necessary to confirm the accuracy and reproducibility of the dispenser. Initial investigation on the accuracy of the dispensed volume was demonstrated using the dipyridamole suspension 
(density= $1.008 \mathrm{~g} / \mathrm{mL}$ ). A linear relationship was established between the theoretical volumes and the actual dispensed volumes from the liquid dispenser across the different sizes of nozzle used with $R^{2}$ values $>0.9992$ (Supplementary Data, Figs. S2 and S3). While a smaller aperture nozzle $(0.35 \mathrm{~mm})$ provided a better accuracy, it it frequently blocked, and a nozzle diameter of $0.41 \mathrm{~mm}$ was used as a default.

The impact of different syringe sizes on the dispensed volumes and dosing accuracy of the liquid dispenser was also investigated (Figs. 7A and 7B). Reducing the size of the syringe used on the liquid dispenser influences both the volume of dispensed liquid and its accuracy. The $2 \mathrm{~mL}$ syringe produced a narrower deviation and was used as a default syringe for further studies. This could be directly related to the mechanism of liquid discharge from the syringe dispenser. During liquid dispensing, although the distance travelled by the piston of the liquid dispenser motor remained identical for both 2 and $10 \mathrm{~mL}$ syringes, however the dispensed volume is significantly reduced by a syringe of smaller diameter. The control of liquid dispensing could also be achieved through the use of pneumatic liquid dispensers (Xie et al., 2010).

Different liquid volumes $(80,160,240$ or $320 \mu \mathrm{L}$ ) of dipyridamole suspension (median particles size $5.08 \pm 0.68 \mu \mathrm{m}$ ) were dispensed and analysed for dosing accuracy by HPLC. It was possible to dictate the dispensed volume and hence corresponding doses by the software modification of the digital core's volume (Fig 8A, Table 2). The linearity between the estimated and the actual dose $\left(\mathrm{R}^{2}=0.9985\right)$ (Fig. 8B) demonstrated the promising potential of this dispenser for on demand drug dosing. The accuracy of dosing was affected by surface tension related phenomena such as foam forming and droplet hanging. These were noted in both printing modes and are potentially could be mitigated in commercial manufacturing via adjustments to the programme for the dispenser motor.

In order to determine the release profile of the 3D printed liquid capsule, a USP II dissolution apparatus was used. Initially, an optimised suspension of dipyridamole was dispensed in a Eudragit EPO shell at different doses. An immediate drug release profile was obtained as $>85$ $\%$ of the active was released before $30 \mathrm{~min}$ (Fig. 9A), and complied with USP criteria for oral dipyridamole products. (Convention, 2007) This might be attributed to rapid ionization of cationic chains of the methacrylate polymer in gastric medium (Sadia et al., 2016) as well as high solubility of dipyridamole in this medium (Alhnan et al., 2010; Paprskarova et al., 2016).

In order to prove the suitability of this capsule system to drug solution, the model core of drug suspension was substituted by a theophylline solution. The shell system was also effective in containing the liquid drug payload without compromising the integrity of the shell, with the absence of leakage during or after printing. In vitro, the capsules demonstrated their ability to 
dissolve quickly leading to a complete release of theophylline before $30 \mathrm{~min}$ (Fig. 9B). This also demonstrated the versatility of the presented approach in the delivery of potentially a wide range of actives in different forms, and potentially hold the promise to be used where thermal processing must be avoided to minimize aggregation and potency loss.

The capacity of this shell system to extend drug release has also been subject to preliminary investigation, employing a water insoluble permeable polymer, Eudragit $R L$ in the filament used to fabricate the shell (Fig. 9C). This is essentially a reservoir-based controlled release system where amongst other factors, formulation of reservoir vehicle, drug loading, drug solubility, drug diffusivity through shell, plasticiser or pore formers in shell can all impact drug release rate (Ahmed and Naini, 2010).

By altering the shell thickness $(1.6,2$ or $2.4 \mathrm{~mm})$, it was possible to control drug release simply through a digital order, without the need to change the formulation composition. It is likely that soluble drug permeates through the insoluble acrylate layer through diffusion mechanism (Evonik, 2010). As the shell thickness increases, the travelled distance of drug will increase too thereby further prolonging drug release. It is worth noting here that the thickness of coating far exceeds typical Eudragit coating systems for pellets (Akhgari and Tavakol, 2016), this might be attributed to the porous nature of 3D printed shell due to voids between fused layers as well as the pores within the filament structure (Tsuda et al., 2015). It is worth noting that conventional coating system can provide a tighter control in comparison to the proposed 3D printed system (Emami and Kazemali, 2016; Siddique et al., 2010). However, this is the first report to achieve such control of drug release from a 3D printed capsule. With on-going advances in additive manufacturing in general, we expect that many of such limitations will be overcome in the future attempts.

In summary, we have reported a dynamic capsule-dispensing platform based on the orchestration of FDM 3D printing and liquid dispensing. Such platform is of high value when providing a small volume of liquid drug payload in a capsule is desirable. It could be potentially developed to include thermolabile substrate in the core, where hot melt extrusion (Patil et al., 2016) and FDM 3D printing (Okwuosa et al., 2016) are usually avoided. Other complex release profiles could also be feasible since the interior of the capsule shell could be compartmentalised with different wall thicknesses, which could be potentially filled with different actives for multiple active administration.

\section{Conclusions}

This is the first report of a fully automated process for the 3D printing of a liquid capsule. Both immediate and extended drug release profiles based on polymethacrylate polymer shells were achieved. Although the capsule shell was constructed in layer-by-layer fashion, it was possible 
to construct a structure that maintains capsule shell integrity and instantly contains the loaded liquid dose without any curing step. The system also proved suitable for two APIs (dipyridamole and theophylline) and for suspensions or solutions as core materials. Above all, this technique demonstrated the ability to control dose as well as drug release by manipulating the dispensed volume and shell thickness simply via software. In a clinical setting, this will empower healthcare staff with capability to provide specific dosing and drug release in individualized liquid capsules without the need to change formulation. 


\section{List of Figures}

Figure 1 Schematic illustration of the fabrication of 3D-printed liquid capsule. A dual head 3D printer was modified by replacing the right-hand nozzle with a syringe dispenser. The FDM nozzle head were loaded with HME processed API-free filament of immediate or extended release properties whilst drug solution or suspension were dispensed using syringe of variable sizes and nozzle diameters.

Figure 2 The co-ordination of FDM nozzle and liquid syringe dispenser is achieved in two different printing modes: i) Single-phase printing: (A1) the core is located in the centre of the cavity of the shell, (A2) Shell printing and capsule filling is achieved by alternation at each layer. (A3) Image of completed shell-core designs with dipyridamole core and Eudragit EPO shell. ii) Multi-phase printing: (B1) the core is located in a median level between the bottom shell $(75 \%)$ and the top shell (25\%). (B2) the shell is printed first followed by complete filling of the shell bottom, the printing is completed by printing the shell top. (B3) Image of completed shell-core designs (shell top was separated from bottom for demonstration). (See Supplementary Data Videos S1 and S2)

Figure 3 (A1 and A2) TGA analysis and (B1 and B2) DSC thermographs of polymer, polymer : TEC physical mixture, polymer : TEC : talc physical mixture, filament and 3D printed shell for Eudragit EPO and $R L$ respectively.

Figure 4 XRD patterns of polymer, talc and polymer: TEC: talc physical mixture, filament and 3D printed shell for Eudragit E.

Figure 5 Impact of 3D printing pattern on the structure and integrity of the shell: SEM images of (A1 and B1) external surface and (A2 and B2) internal surface of the Eudragit $E$ based shell, in rectilinear or concentric infill respectively.

Figure 6 The impact of printing pattern on the structure of the shell: SEM image of external surface of the corner and side of Eudragit $E$ based capsule shell produced via (A1 and A2) single-phase or (B1 and B2) multi-phase 3D printing.

Figure 7 Impact of single-phase and multi-phase 3D printing modes on dispensed volume of dipyridamole suspension (1.5\% w/v) from the liquid dispenser using (A) $2 \mathrm{~mL}$ or (B) $10 \mathrm{~mL}$ syringe.

Figure 8 Dose accuracy using syringe liquid dispenser of dipyridamole suspension $(1.5 \% \mathrm{w} / \mathrm{v})$ : (A) relation of theoretical volume of the core in the software and dispensed dose using singleor multi-phase 3D printing modes, (B) correlation between theoretical and actual volume. 
Figure 9 A) In vitro release pattern of dipyridamole suspension from 3D printed liquid Eudragit EPO capsule using USP II with different core volumes in gastric media ( $\mathrm{pH}$ 1.2). B) In vitro release of theophylline from 3D printed liquid capsule filled with theophylline solution based on (B) Eudragit $E$ or $(C)$ Eudragit RL. All capsules were printed using multi-phase mode 3D printing and $2 \mathrm{~mL}$ syringe liquid dispenser.

\section{List of Tables}

Table 1 The formulation and processing parameters for HME and FDM 3D printing of shell filament formulations

Table 2 A summary of the volume, dimensions and respective volumes, estimated volume, estimated dose and actual dose for different cores in the 3D printed liquid capsules $(n=4$, $\pm S D)$.

\section{Supplementary Data}

Figure S1 Modification of dual FDM 3D printer to accommodate a liquid dispenser (right) in combination with FDM 3D printer head (left).

Figure S2 Linear relationship theoretical volume of the software and dispensed volume using single-phase printing mode and different nozzle aperture sizes (blue, orange and grey graphs for $0.25,0.41$ or $0.84 \mathrm{~mm}$ nozzles respectively).

Figure S3 Linear relationship theoretical volume of the software and dispensed volume using multi-phase printing mode and different nozzle aperture sizes (blue, orange and grey graphs for $0.25,0.41$ or $0.84 \mathrm{~mm}$ nozzles respectively).

Video S1 Single-phase 3D printing of liquid Eudragit capsule filled with dipyridamole suspensions.

Video S2 Multi-phase 3D printing of liquid Eudragit capsule filled with dipyridamole suspensions. 


\section{References}

Ahmed, S.U., Naini, V., 2010. Generic Oral Controlled Release Product Development: Formulation and Process Considerations, in: Wen, H., Park, K. (Eds.), Oral Controlled Release Formulation Design and Drug Delivery. John Wiley \& Sons, Inc., New Jersy, pp. 321-335.

Akhgari, A., Tavakol, A., 2016. Prediction of Optimum Combination of Eudragit RS/Eudragit RL/Ethyl Cellulose Polymeric Free Films Based on Experimental Design for Using as a Coating System for Sustained Release Theophylline Pellets. Adv Pharm Bull 6, 219-225.

Al-Metwali, B., Mulla, H., 2017. Personalised dosing of medicines for children. J Pharm Pharmacol 69, 514-524.

Alhnan, M.A., Cosi, D., Murdan, S., Basit, A.W., 2010. Inhibiting the gastric burst release of drugs from enteric microparticles: the influence of drug molecular mass and solubility. J Pharm Sci 99, 4576-4583. Alhnan, M.A., Okwuosa, T.C., Sadia, M., Wan, K.-W., Ahmed, W., Arafat, B., 2016. Emergence of 3D Printed Dosage Forms: Opportunities and Challenges. Pharm Res 33, 1817-1832.

Armstrong, N.A., James, K.C., Pugh, W.K., 1984. Drug migration into soft gelatin capsule shells and its effect on in-vitro availability. J Pharm Pharmacol 36, 361-365.

Ashour, E.A., Majumdar, S., Alsheteli, A., Alshehri, S., Alsulays, B., Feng, X., Gryczke, A., Kolter, K., Langley, N., Repka, M.A., 2016. Hot melt extrusion as an approach to improve solubility, permeability and oral absorption of a psychoactive natural product, piperine. J Pharm Pharmacol 68, 989-998.

Capsugel, 2012. A New HPMC Capsule for Optimum Formulation pf Pharmaceutical Dosage Forms. Chai, X., Chai, H., Wang, X., Yang, J., Li, J., Zhao, Y., Cai, W., Tao, T., Xiang, X., 2017. Fused Deposition Modeling (FDM) 3D Printed Tablets for Intragastric Floating Delivery of Domperidone. Sci Rep 7, 2829. Clark, E.A., Alexander, M.R., Irvine, D.J., Roberts, C.J., Wallace, M.J., Sharpe, S., Yoo, J., Hague, R.J.M., Tuck, C.J., Wildman, R.D., 2017. 3D printing of tablets using inkjet with UV photoinitiation. Int J Pharm 529, 523-530.

Cole, E.T., Cadé, D., Benameur, H., 2008. Challenges and opportunities in the encapsulation of liquid and semi-solid formulations into capsules for oral administration. Adv Drug Deliv Rev 60, 747-756.

Convention, U.S.P., 2007. The United States Pharmacopeia : USP30 : the National Formulary : NF25. United States Pharmacopeial Convention Inc., Rockville, Md.

Dave, V.S., Fahmy, R.M., Hoag, S.W., 2015. Near-infrared spectroscopic analysis of the breaking force of extended-release matrix tablets prepared by roller-compaction: influence of plasticizer levels and sintering temperature. Drug Dev Ind Pharm 41, 898-905.

Deepthi, Y., Murthy, T.E.G., 2015. Design and development and evaluation of candesartan cilexetil liquid filling formulations. Int J Pharm Investig 5, 81-86.

Elzayat, E.M., Abdel-Rahman, A.A., Ahmed, S.M., Alanazi, F.K., Habib, W.A., Abou-Auda, H.S., Sakr, A., 2017. Formulation and pharmacokinetics of multi-layered matrix tablets: Biphasic delivery of diclofenac. Saudi Pharm J 25, 688-695.

Emami, J., Kazemali, M.R., 2016. Design and in vitro evaluation of a novel controlled onset extendedrelease delivery system of metoprolol tartrate. Res Pharm Sci 11, 81-92.

Evonik, 2010. Methacrylate Polymers for Oral Drug Delivery.

Goyanes, A., Chang, H., Sedough, D., Hatton, G.B., Wang, J., Buanz, A., Gaisford, S., Basit, A.W., 2015. Fabrication of controlled-release budesonide tablets via desktop (FDM) 3D printing. Int J Pharm 496, 414-420.

Goyanes, A., Fina, F., Martorana, A., Sedough, D., Gaisford, S., Basit, A.W., 2017. Development of modified release 3D printed tablets (printlets) with pharmaceutical excipients using additive manufacturing. Int J Pharm 527, 21-30.

Gross, B.C., Erkal, J.L., Lockwood, S.Y., Chen, C., Spence, D.M., 2014. Evaluation of 3D Printing and Its Potential Impact on Biotechnology and the Chemical Sciences. Anal Chem 86, 3240-3253.

Higashi, K., Seo, A., Egami, K., Otsuka, N., Limwikrant, W., Yamamoto, K., Moribe, K., 2016. Mechanistic insight into the dramatic improvement of probucol dissolution in neutral solutions by solid dispersion in Eudragit E PO with saccharin. J Pharm Pharmacol 68, 655-664. 
Hom, F.S., Veresh, S.A., Ebert, W.R., 1975. Soft gelatin capsules II: Oxygen permeability study of capsule shells. J Pharm Sci 64, 851-857.

Hussein, A., El-Menshawe, S., Afouna, M., 2012. Enhancement of the in-vitro dissolution and in-vivo oral bioavailability of silymarin from liquid-filled hard gelatin capsules of semisolid dispersion using Gelucire 44/14 as a carrier. Die Pharmazie 67, 209-214.

Kyobula, M., Adedeji, A., Alexander, M.R., Saleh, E., Wildman, R., Ashcroft, I., Gellert, P.R., Roberts, C.J., 2017. 3D inkjet printing of tablets exploiting bespoke complex geometries for controlled and tuneable drug release. J Control Release 261, 207-215.

Li, S., Tian, Y., Jones, D.S., Andrews, G.P., 2016a. Optimising Drug Solubilisation in Amorphous Polymer Dispersions: Rational Selection of Hot-melt Extrusion Processing Parameters. AAPS PharmSciTech 17, 200-213.

Li, S., Yu, T., Tian, Y., McCoy, C.P., Jones, D.S., Andrews, G.P., 2016b. Mechanochemical Synthesis of Pharmaceutical Cocrystal Suspensions via Hot Melt Extrusion: Feasibility Studies and Physicochemical Characterization. Mol Pharm 13, 3054-3068.

Limited, E.P., 2014. Specifications For Empty Hard Gelatin Capsules

Lucas, R.A., Bowtle, W.J., Ryden, R., 1987. Disposition of vancomycin in healthy volunteers from oral solution and semi-solid matrix capsules. J Clin Pharm Ther 12, 27-31.

Markl, D., Zeitler, J.A., Rasch, C., Michaelsen, M.H., Müllertz, A., Rantanen, J., Rades, T., Bøtker, J., 2017. Analysis of 3D Prints by X-ray Computed Microtomography and Terahertz Pulsed Imaging. Pharm Res 34, 1037-1052.

McDougall, D.A.J., Martin, J., Playford, E.G., Green, B., 2016. Determination of a suitable voriconazole pharmacokinetic model for personalised dosing. J Pharmacokinet Pharmacodyn 43, 165-177.

Melocchi, A., Parietti, F., Loreti, G., Maroni, A., Gazzaniga, A., Zema, L., 2015. 3D printing by fused deposition modeling (FDM) of a swellable/erodible capsular device for oral pulsatile release of drugs. J Drug Deliv Sci Technol.

Ochi, M., Kimura, K., Kanda, A., Kawachi, T., Matsuda, A., Yuminoki, K., Hashimoto, N., 2016. Physicochemical and Pharmacokinetic Characterization of Amorphous Solid Dispersion of Meloxicam with Enhanced Dissolution Property and Storage Stability. AAPS PharmSciTech 17, 932-939.

Okwuosa, T.C., Pereira, B.C., Arafat, B., Cieszynska, M., Isreb, A., Alhnan, M.A., 2017. Fabricating a Shell-Core Delayed Release Tablet Using Dual FDM 3D Printing for Patient-Centred Therapy. Pharm Res 34, 427-437.

Okwuosa, T.C., Stefaniak, D., Arafat, B., Isreb, A., Wan, K.-W., Alhnan, M.A., 2016. A Lower Temperature FDM 3D Printing for the Manufacture of Patient-Specific Immediate Release Tablets. Pharm Res 33, 2704-2712.

Ozguney, I., Ozcan, I., Ertan, G., Guneri, T., 2007. The preparation and evaluation of sustained release suppositories containing ketoprofen and Eudragit RL 100 by using factorial design. Pharm Dev Technol 12, 97-107.

Paprskarova, A., Mozna, P., Oga, E.F., Elhissi, A., Alhnan, M.A., 2016. Instrumentation of Flow-Through USP IV Dissolution Apparatus to Assess Poorly Soluble Basic Drug Products: a Technical Note. AAPS PharmSciTech 17, 1261-1266.

Park, K., 2015. 3D printing of 5-drug polypill. J Control Release 217, 352.

Patil, H., Tiwari, R.V., Repka, M.A., 2016. Hot-Melt Extrusion: from Theory to Application in Pharmaceutical Formulation. AAPS PharmSciTech 17, 20-42.

Pietrzak, K., Isreb, A., Alhnan, M.A., 2015. A flexible-dose dispenser for immediate and extended release 3D printed tablets. Eur J Pharm Biopharm.

Qiao, M., Zhang, L., Ma, Y., Zhu, J., Chow, K., 2010. A novel electrostatic dry powder coating process for pharmaceutical dosage forms: immediate release coatings for tablets. Eur J Pharm Biopharm 76, 304-310.

Ryu, G.S., Lee, Y.J., 2012. Analysis of liquid medication dose errors made by patients and caregivers using alternative measuring devices. J Manag Care Pharm 18, 439-445. 
Saal, W., Ross, A., Wyttenbach, N., Alsenz, J., Kuentz, M., 2017. A Systematic Study of Molecular Interactions of Anionic Drugs with a Dimethylaminoethyl Methacrylate Copolymer Regarding Solubility Enhancement. Mol Pharm 14, 1243-1250.

Saal, W., Ross, A., Wyttenbach, N., Alsenz, J., Kuentz, M., 2018. Unexpected Solubility Enhancement of Drug Bases in the Presence of a Dimethylaminoethyl Methacrylate Copolymer. Mol Pharm 15, 186192.

Sadia, M., Sosnicka, A., Arafat, B., Isreb, A., Ahmed, W., Kelarakis, A., Alhnan, M.A., 2016. Adaptation of pharmaceutical excipients to FDM 3D printing for the fabrication of patient-tailored immediate release tablets. Int J Pharm 513, 659-668.

Siddique, S., Khanam, J., Bigoniya, P., 2010. Development of sustained release capsules containing "coated matrix granules of metoprolol tartrate". AAPS PharmSciTech 11, 1306-1314.

Skowyra, J., Pietrzak, K., Alhnan, M.A., 2015. Fabrication of extended-release patient-tailored prednisolone tablets via fused deposition modelling (FDM) 3D printing. Eur J Pharm Sci 68, 11-17.

Sobhani, P., Christopherson, J., Ambrose, P.J., Corelli, R.L., 2008. Accuracy of oral liquid measuring devices: comparison of dosing cup and oral dosing syringe. Ann Pharmacother 42, 46-52.

Taki, M., Tagami, T., Ozeki, T., 2017. Preparation of polymer-blended quinine nanocomposite particles by spray drying and assessment of their instrumental bitterness-masking effect using a taste sensor. Drug Dev Ind Pharm 43, 715-722.

Thingiverse, 2017. Thingyverse website (https://www.thingiverse.com/thing:20733) last accessed 23/6/2017.

Tsuda, S., Jaffery, H., Doran, D., Hezwani, M., Robbins, P.J., Yoshida, M., Cronin, L., 2015. Customizable 3D Printed 'Plug and Play' Millifluidic Devices for Programmable Fluidics. Plos One 10.

Xie, D., Zhang, H., Shu, X., Xiao, J., Cao, S., 2010. Multi-materials drop-on-demand inkjet technology based on pneumatic diaphragm actuator. Sci China Tech Sci 53, 1605-1611.

Yang, Q., Ma, Y., Zhu, J., 2015. Applying a novel electrostatic dry powder coating technology to pellets. Eur J Pharm Biopharm 97, 118-124. 


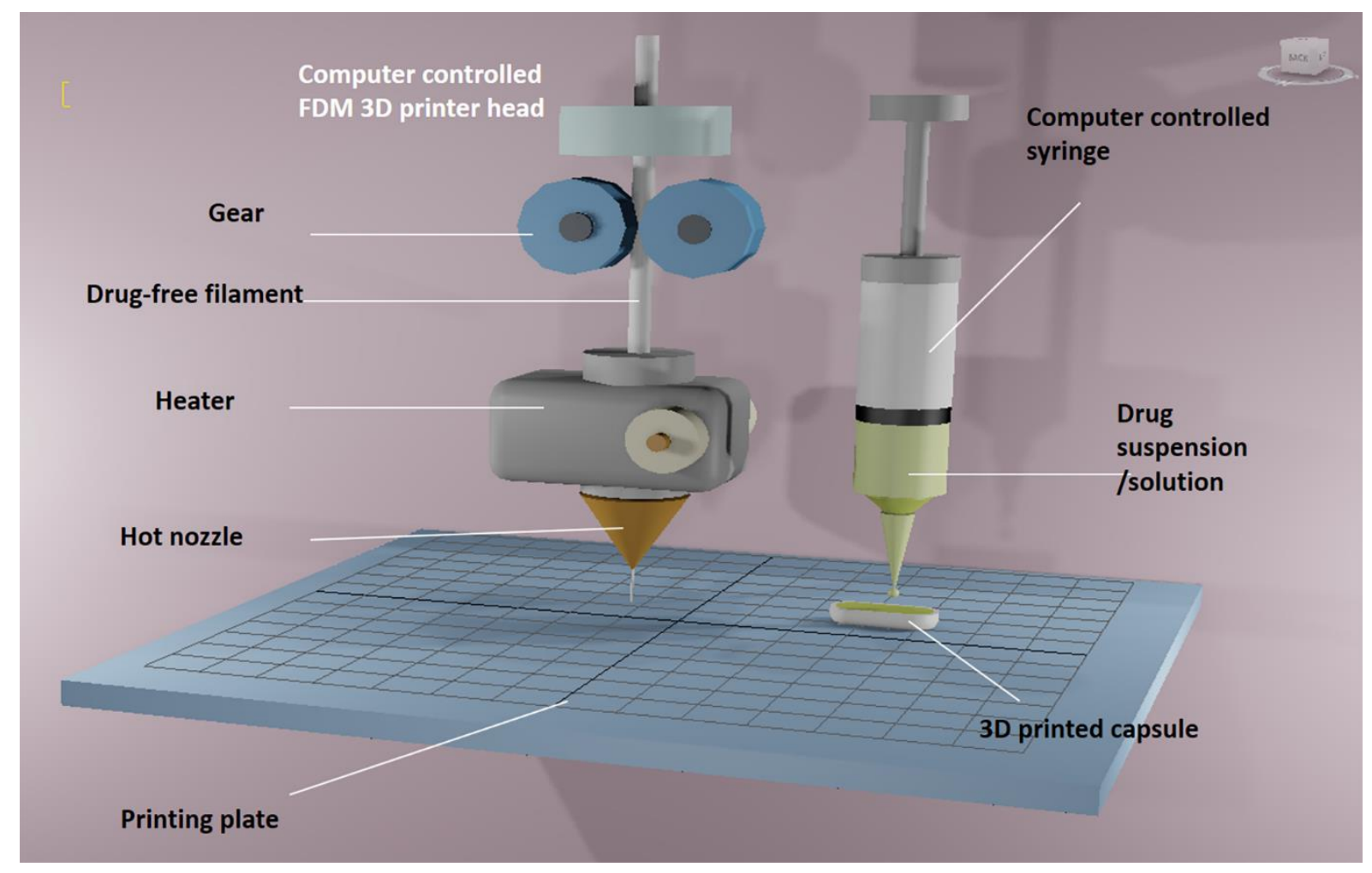

Figure 1 Schematic illustration of the fabrication of 3D-printed liquid capsule. A dual head 3D printer was modified by replacing the right-hand nozzle with a syringe dispenser. The FDM nozzle head were loaded with HME processed API-free filament of immediate or extended release properties whilst drug solution or suspension were dispensed using syringe of variable sizes and nozzle diameters. 

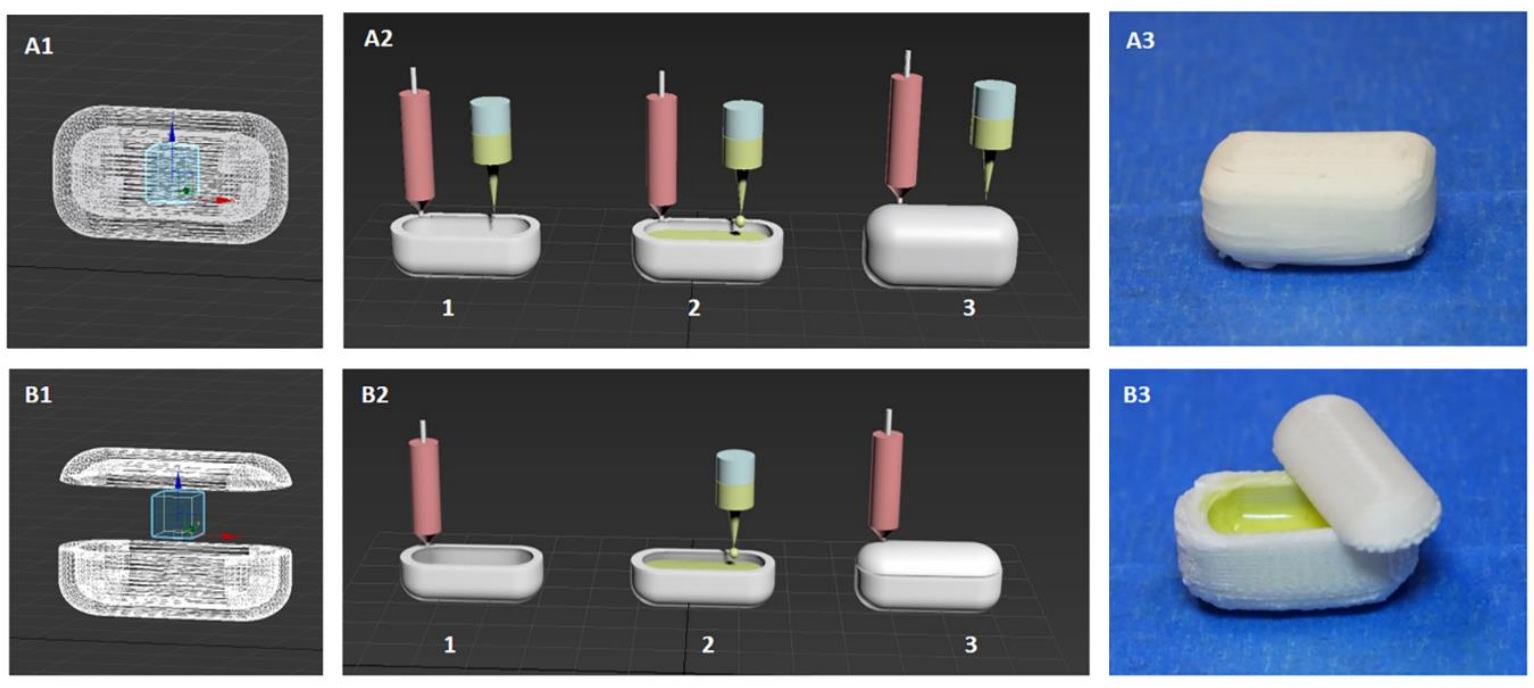

Figure 2 The co-ordination of FDM nozzle and liquid syringe dispenser is achieved in two different printing modes: i) Single-phase printing: (A1) the core is located in the centre of the cavity of the shell, (A2) Shell printing and capsule filling is achieved by alternation at each layer. (A3) Image of completed shell-core designs with dipyridamole core and Eudragit EPO shell. ii) Multi-phase printing: (B1) the core is located in a median level between the bottom shell (75\%) and the top shell (25\%). (B2) the shell is printed first followed by complete filling of the shell bottom, the printing is completed by printing the shell top. (B3) Image of completed shell-core designs (shell top was separated from bottom for demonstration). (See Supplementary Data Videos S1 and S2) 
A1

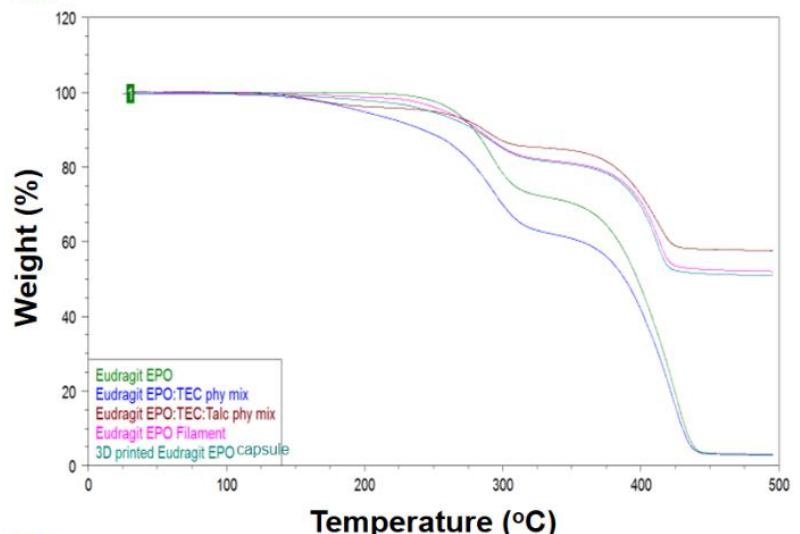

A2

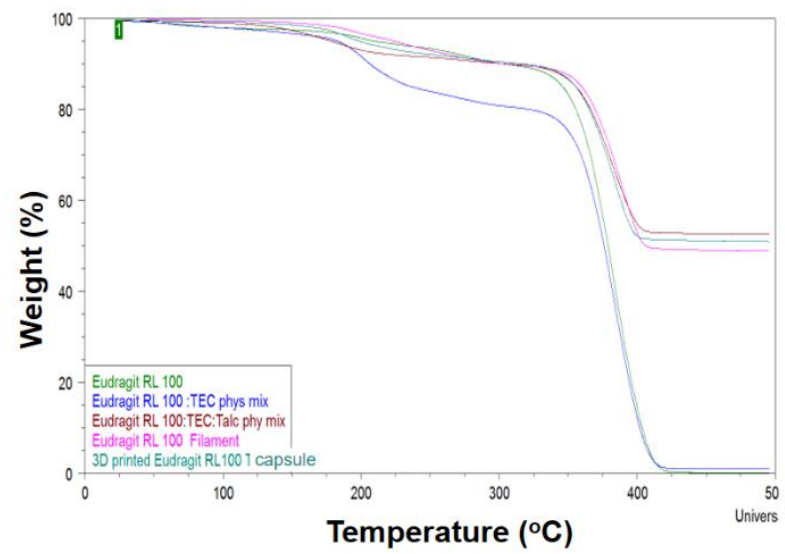

B1

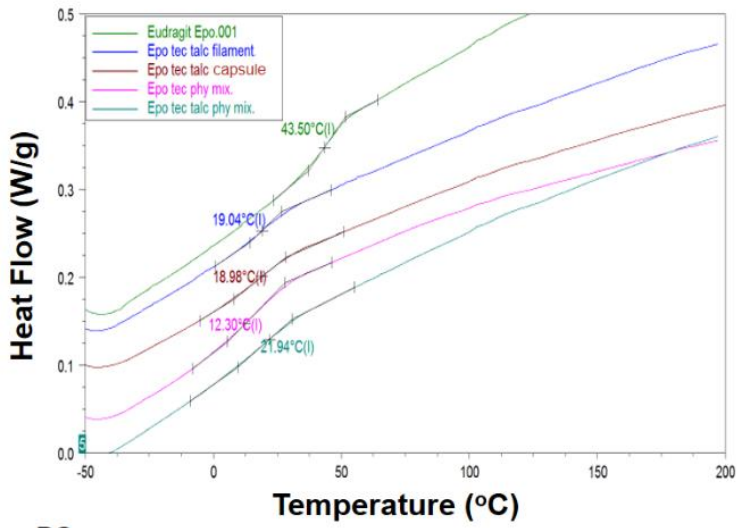

B2

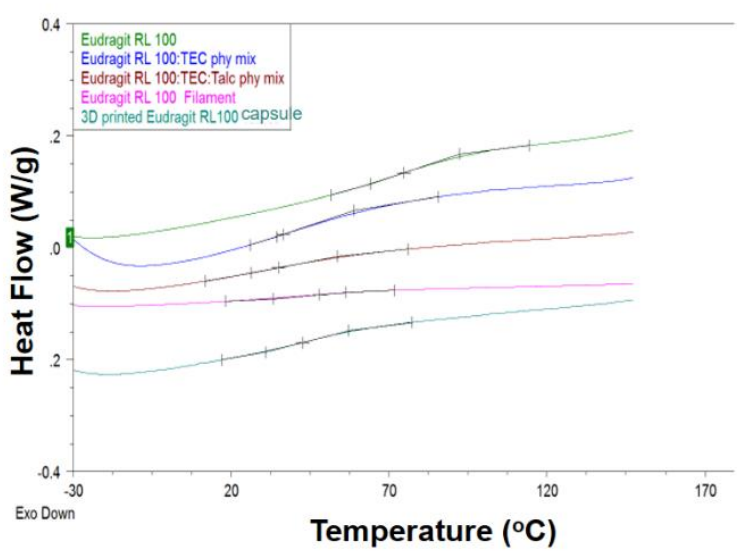

Figure 3 (A1 and A2) TGA analysis and (B1 and B2) DSC thermographs of polymer, polymer : TEC physical mixture, polymer : TEC : talc physical mixture, filament and 3D printed shell for Eudragit EPO and RL respectively. 


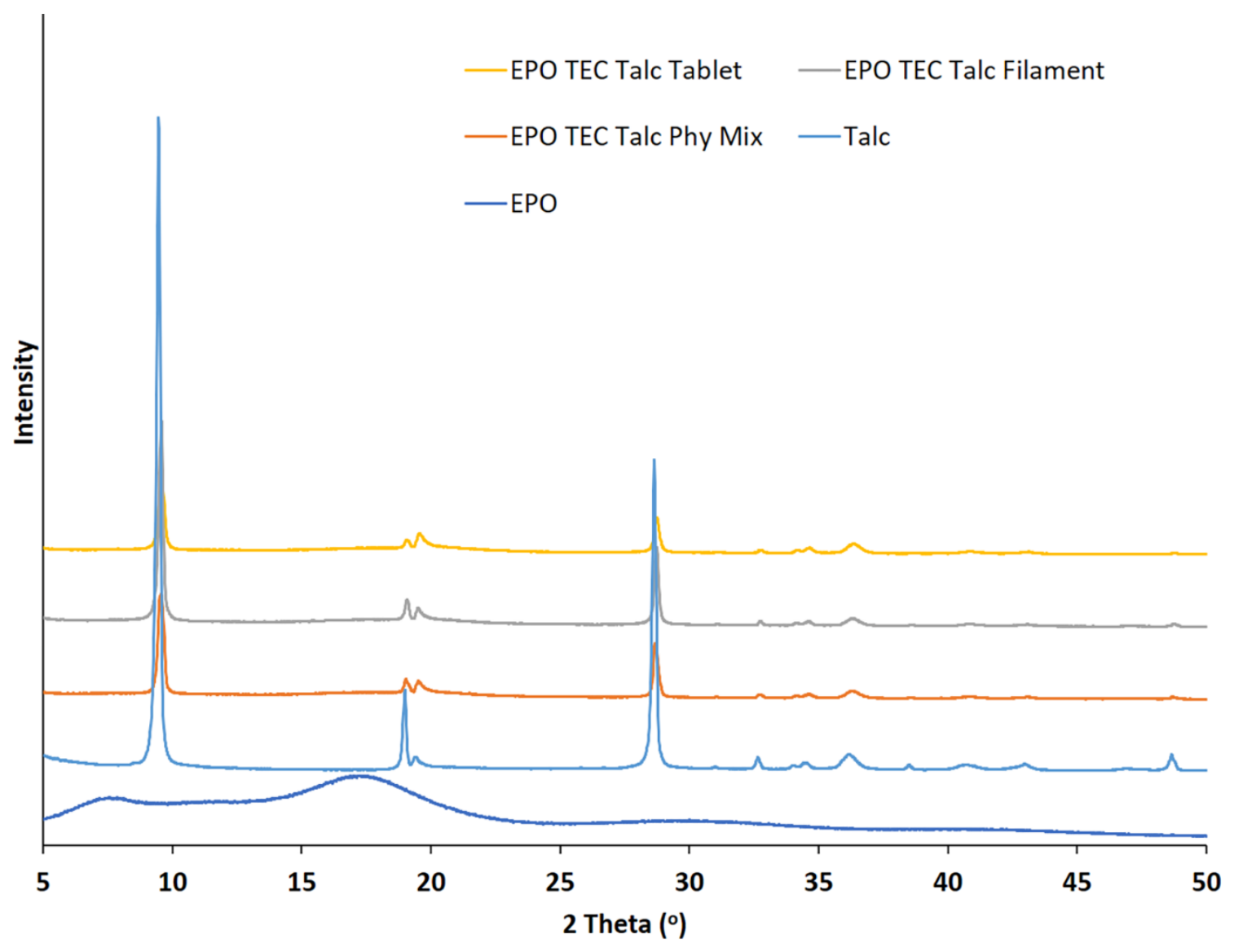

Figure 4 XRD patterns of polymer, talc and polymer: TEC: talc physical mixture, filament and 3D printed shell for Eudragit E. 

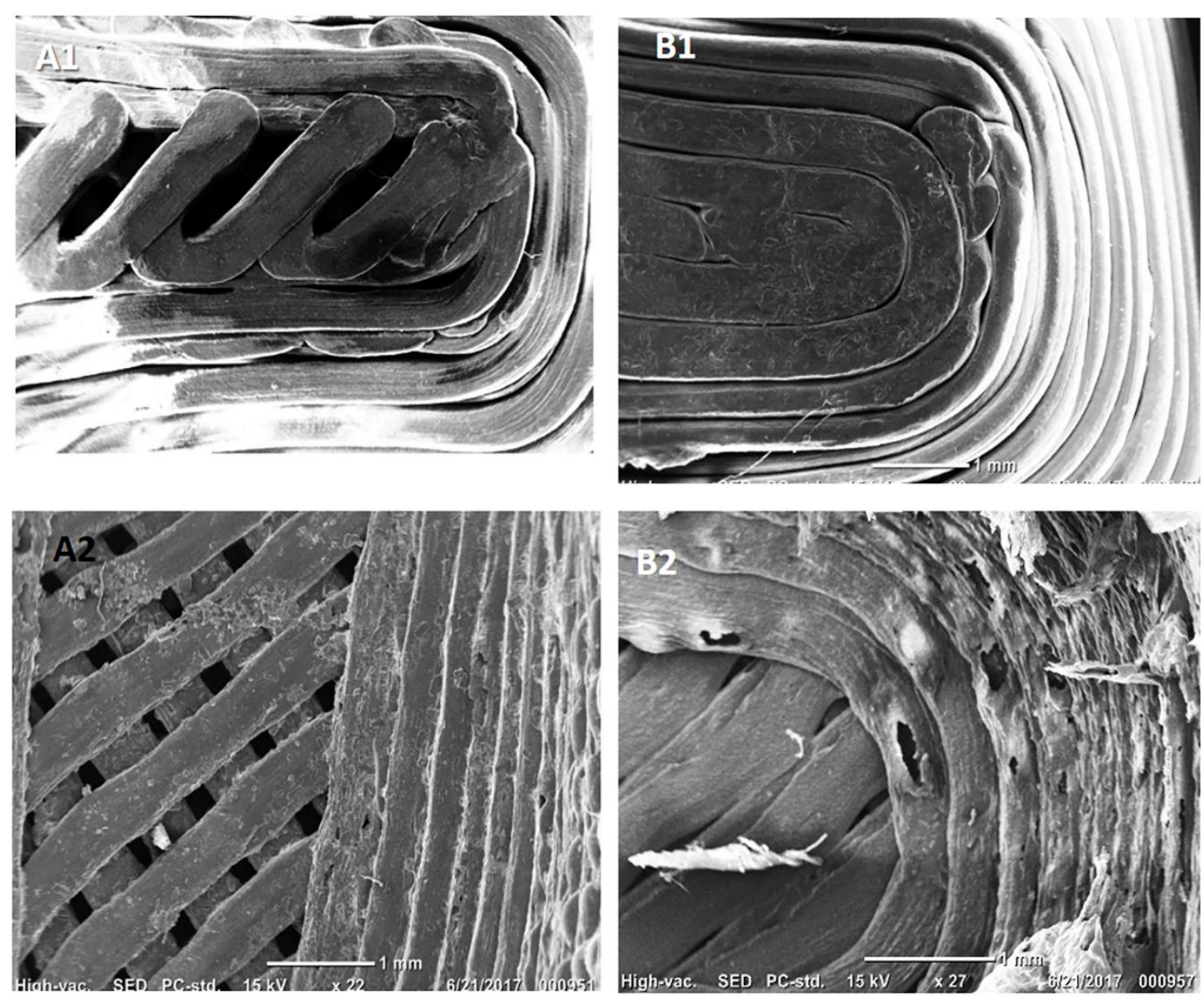

Figure 5 Impact of 3D printing pattern on the structure and integrity of the shell: SEM images of (A1 and B1) external surface and (A2 and B2) internal surface of the Eudragit $E$ based shell, in rectilinear or concentric infill respectively. 

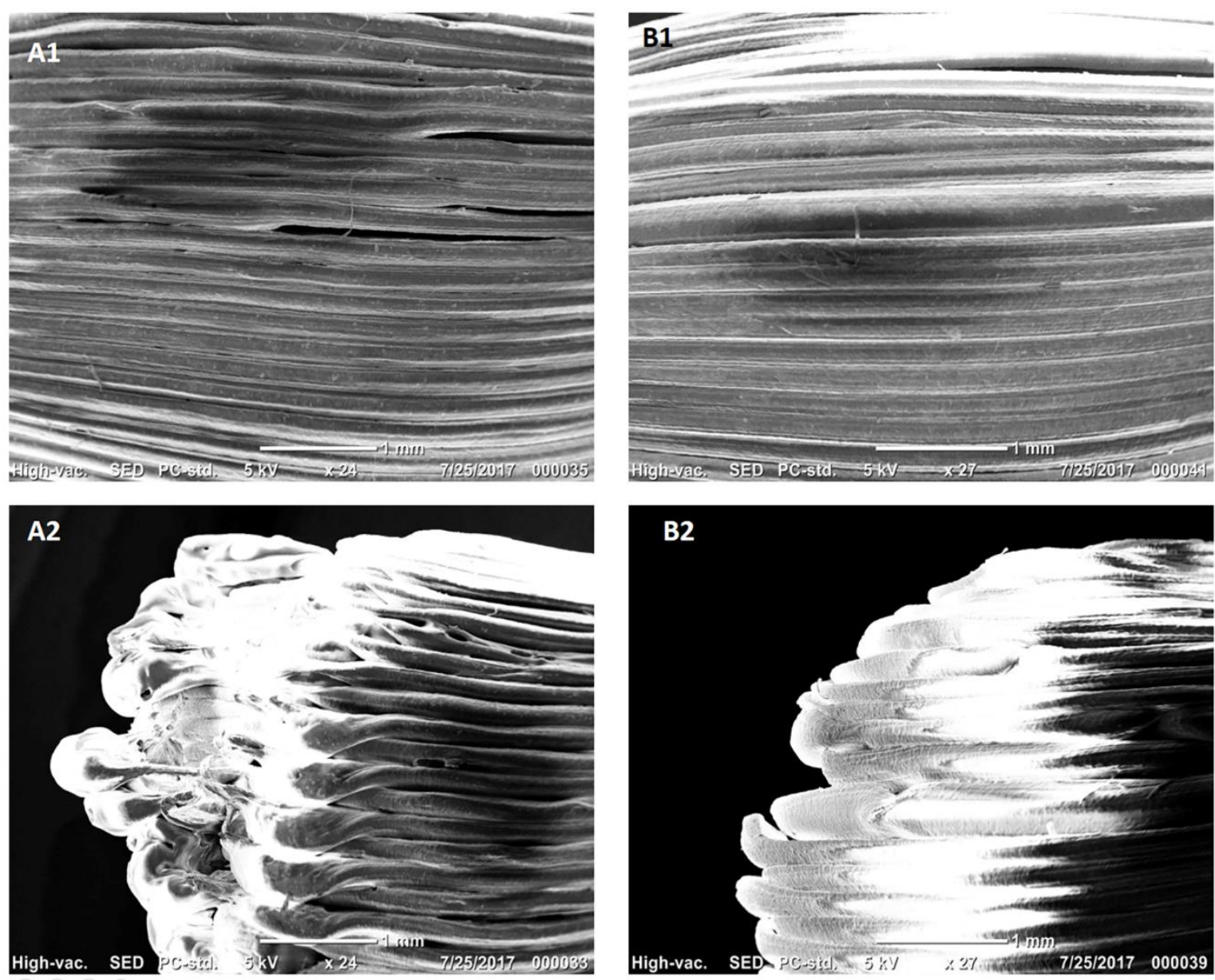

Figure 6 The impact of printing pattern on the structure of the shell: SEM image of external surface of the corner and side of Eudragit $E$ based capsule shell produced via (A1 and A2) single-phase or (B1 and B2) multi-phase 3D printing. 
A

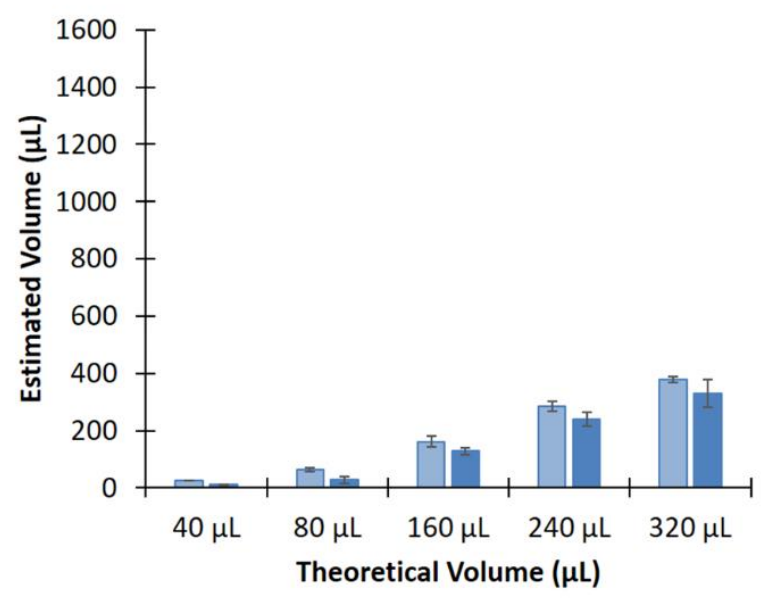

$\square 2 \mathrm{~mL}$ Syringe Single-Phase Printing

- $2 \mathrm{~mL}$ Syringe Multi-Phase Printing
B

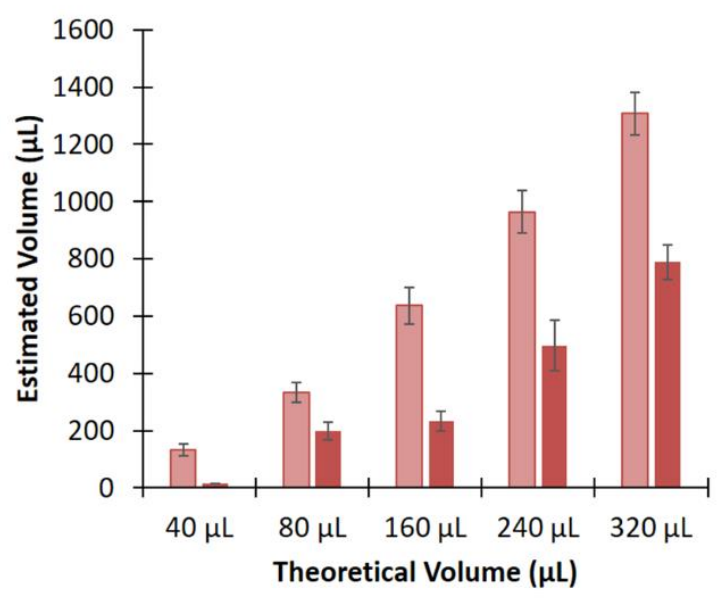

$\square 10 \mathrm{~mL}$ Syringe Single-Phase Printing

a $10 \mathrm{~mL}$ Syringe Multi-Phase Printing

Figure 7 Impact of single-phase and multi-phase 3D printing modes on dispensed volume of dipyridamole suspension (1.5\% w/v) from the liquid dispenser using (A) $2 \mathrm{~mL}$ or (B) $10 \mathrm{~mL}$ syringe. 
A

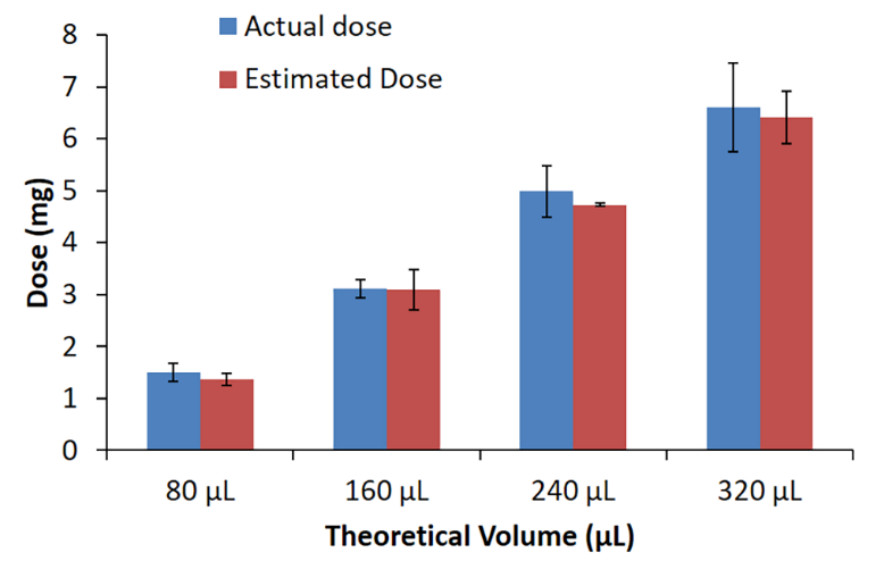

B

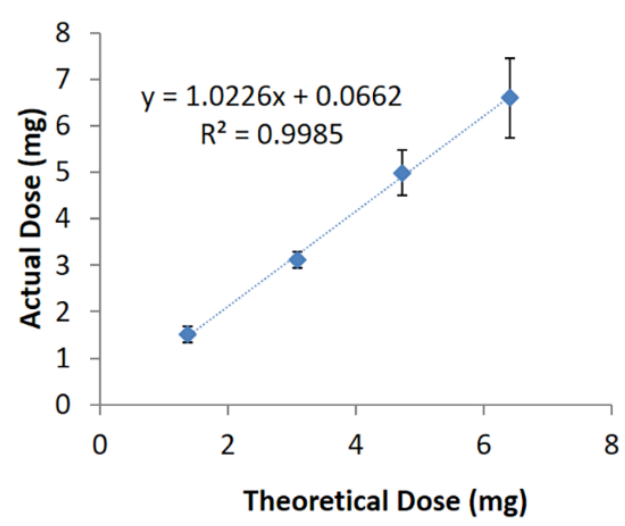

Figure 8 Dose accuracy using syringe liquid dispenser of dipyridamole suspension $(1.5 \% \mathrm{w} / \mathrm{v})$ : (A) relation of theoretical volume of the core in the software and dispensed dose using singleor multi-phase 3D printing modes, (B) correlation between theoretical and actual volume. 
A

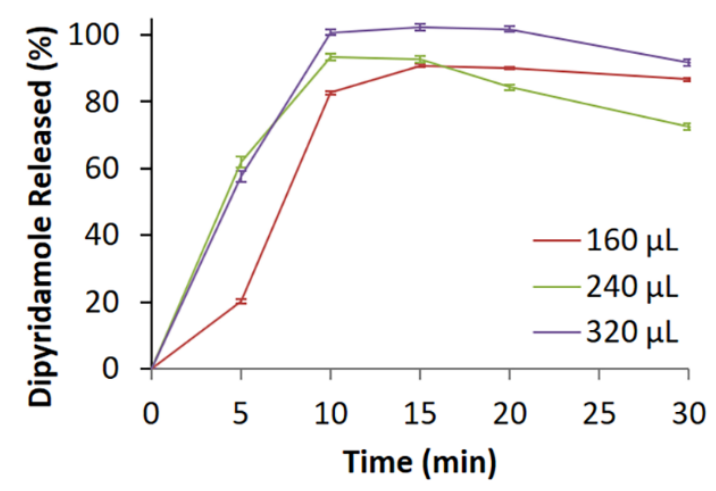

B

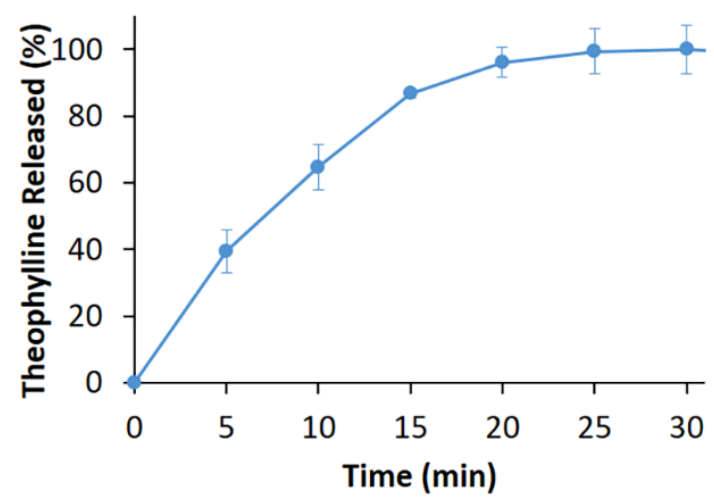

C

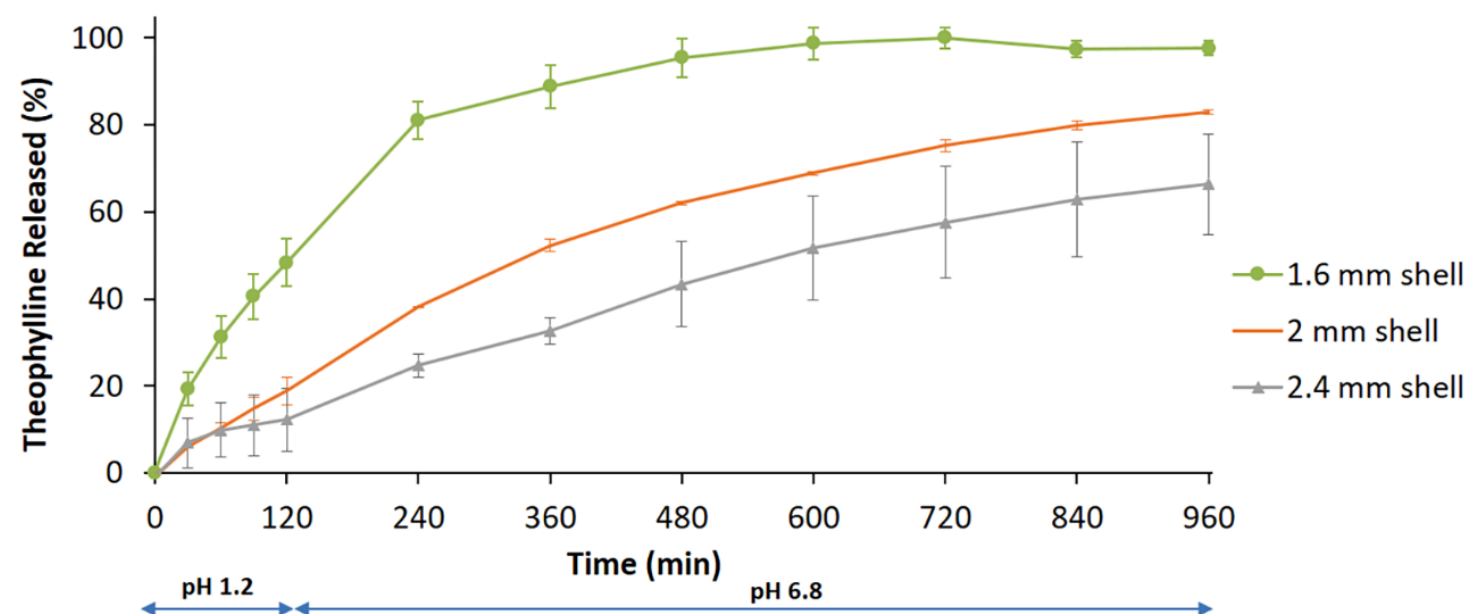

Figure 9 A) In vitro release pattern of dipyridamole suspension from 3D printed liquid Eudragit EPO capsule using USP II with different core volumes in gastric media ( $\mathrm{pH}$ 1.2). B) In vitro release of theophylline from $3 \mathrm{D}$ printed liquid capsule filled with theophylline solution based on (B) Eudragit $E$ or (C) Eudragit RL. All capsules were printed using multi-phase mode 3D printing and $2 \mathrm{~mL}$ syringe liquid dispenser. 
Table 1 The formulation and processing parameters for HME and FDM 3D printing of shell filament formulations

\begin{tabular}{|c|c|c|c|c|c|c|c|c|}
\hline & Polymer (\%) & TEC (\%) & Talc (\%) & $\begin{array}{l}\text { Processing } \\
\text { temperature } \\
\left({ }^{\circ} \mathrm{C}\right)\end{array}$ & $\begin{array}{l}\text { Extrusion } \\
\text { temperature } \\
\left({ }^{\circ} \mathrm{C}\right)\end{array}$ & $\begin{array}{l}\text { Nozzle size } \\
(\mathrm{mm})\end{array}$ & $\begin{array}{l}\text { 3D printing } \\
\text { temperature } \\
\left({ }^{\circ} \mathrm{C}\right)\end{array}$ & $\begin{array}{l}\text { Platform } \\
\text { temperature } \\
\left({ }^{\circ} \mathrm{C}\right)\end{array}$ \\
\hline Eudragit RL & 45 & 5 & 50 & 130 & 120 & 1.7 & 170 & 20 \\
\hline
\end{tabular}


Table 2 A summary of the volume, dimensions and respective volumes, estimated volume, estimated dose and actual dose for different cores in the 3D printed liquid capsules $(n=4, \pm S D)$.

\begin{tabular}{|c|c|c|c|c|c|c|c|c|c|c|c|c|}
\hline \multirow[t]{2}{*}{ Sample } & \multirow[t]{2}{*}{$\begin{array}{l}\text { Theoretical } \\
\text { Volume }(\mu \mathrm{L})\end{array}$} & \multicolumn{3}{|c|}{$\begin{array}{l}\text { Core's Dimensions } \\
(\mathrm{mm})\end{array}$} & \multicolumn{3}{|c|}{$\begin{array}{l}\text { Shell's Dimensions } \\
\text { (mm) }\end{array}$} & \multirow[t]{2}{*}{$\begin{array}{l}\text { Core weight } \\
\text { (mg) }\end{array}$} & \multirow{2}{*}{$\begin{array}{l}\text { Estimated } \\
\text { dispensed } \\
\text { volume* }(\mu \mathrm{L})\end{array}$} & \multirow[t]{2}{*}{$\begin{array}{l}\text { Estimated } \\
\text { Dose** }^{*}(\mathrm{mg})\end{array}$} & \multirow[t]{2}{*}{$\begin{array}{l}\text { Actual dose }{ }^{\dagger} \\
(\mathrm{mg})\end{array}$} & \multirow{2}{*}{$\begin{array}{l}\text { Dosing } \\
\text { efficiency } \\
(\%)\end{array}$} \\
\hline & & $\mathbf{x}$ & $\mathbf{y}$ & $\mathbf{z}$ & $\mathbf{x}$ & $\mathbf{y}$ & $\mathbf{z}$ & & & & & \\
\hline Core 1 & 80 & 4.32 & 4.32 & 4.32 & 23 & 10.35 & 6.74 & $82.3 \pm 6.95$ & $82.7 \pm 7.0$ & $1.4 \pm 0.1$ & $1.51 \pm 0.2$ & $91.1 \pm 7.5$ \\
\hline Core 2 & 160 & 5.43 & 5.43 & 5.43 & 23 & 10.35 & 6.74 & $185.8 \pm 23.75$ & $186.8 \pm 23.9$ & $3.1 \pm 0.4$ & $3.11 \pm 0.2$ & $99.4 \pm 13.7$ \\
\hline Core 3 & 240 & 6.22 & 6.22 & 6.22 & 23 & 10.35 & 7.74 & $284.6 \pm 1.48$ & $286.1 \pm 1.5$ & $4.7 \pm 0.02$ & $4.99 \pm 0.5$ & $95.6 \pm 9.8$ \\
\hline Core 4 & 320 & 6.84 & 6.84 & 6.84 & 23 & 10.35 & 9.74 & $385.7 \pm 30.57$ & $387.8 \pm 30.7$ & $6.4 \pm 0.5$ & $6.60 \pm 0.9$ & $99.0 \pm 11.5$ \\
\hline
\end{tabular}

*The estimated dispensed volume was calculated based on the density of the dispensed dipyridamole suspension. ${ }^{* *}$ The estimated dose was calculated based on the concentration of the suspension and the estimated dispensed volume. ${ }^{\dagger}$ Actual dose was measured using HPLC, and $\neq$ dosing efficiency was calculated as efficiency $(\%)=($ estimated dose/ actual dose $) \times 100$. 


\section{On Demand Manufacturing of Patient-Specific Liquid Capsules via co-ordinated 3D Printing and Liquid Dispensing}

\section{Supplementary data}

Tochukwu C Okwuosa ${ }^{1}$, Cindy Soares ${ }^{1}$, Verena Golwitzer ${ }^{1,2}$, Rober Habashy ${ }^{1}$, Peter Timmins $^{3}$, Mohamed A Alhnan ${ }^{1}$

${ }^{1}$ School of Pharmacy and Biomedical Sciences, University of Central Lancashire, Preston, Lancashire, UK.

2 Department of Chemistry and Pharmacy, Friedrich-Alexander University ErlangenNürnberg, Erlangen, Germany.

${ }^{3}$ Department of Pharmacy, University of Huddersfield, Huddersfield, UK.

*Corresponding author: MAlbedAlhnan@uclan.ac.uk 


\section{List of figures:}

Figure S1 Modification of dual FDM 3D printer to accommodate a liquid dispenser (right) in combination with FDM 3D printer head (left).

Figure S2 Linear relationship theoretical volume of the software and dispensed volume using single-phase printing mode and different nozzle aperture sizes (blue, orange and grey graphs for $0.25,0.41$ or $0.84 \mathrm{~mm}$ nozzles respectively).

Figure S3 Linear relationship theoretical volume of the software and dispensed volume using multi-phase printing mode and different nozzle aperture sizes (blue, orange and grey graphs for $0.25,0.41$ or $0.84 \mathrm{~mm}$ nozzles respectively).

\section{List of Videos:}

Video S1 Single phase 3D printing of liquid Eudragit capsule filled with dipyridamole suspensions.

Video S2 Multi-phase 3D printing of liquid Eudragit capsule filled with dipyridamole suspensions. 


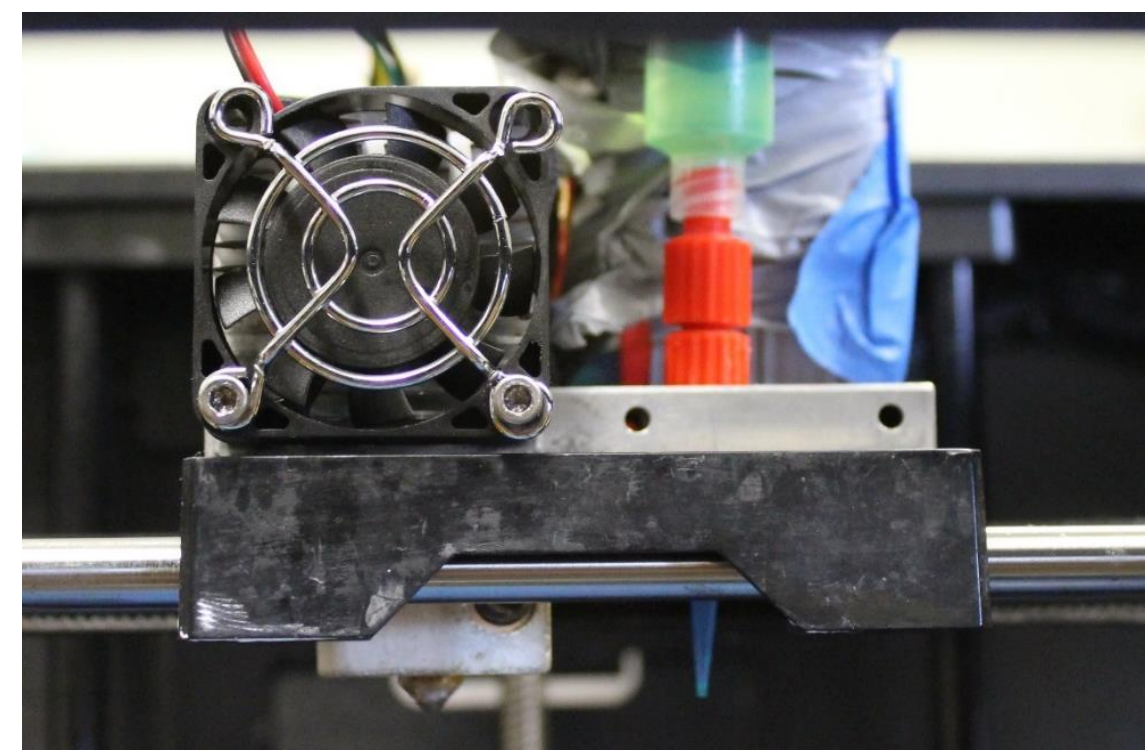

Figure S1 Modification of dual FDM 3D printer to accommodate a liquid dispenser (right) in combination with FDM 3D printer head (left). 

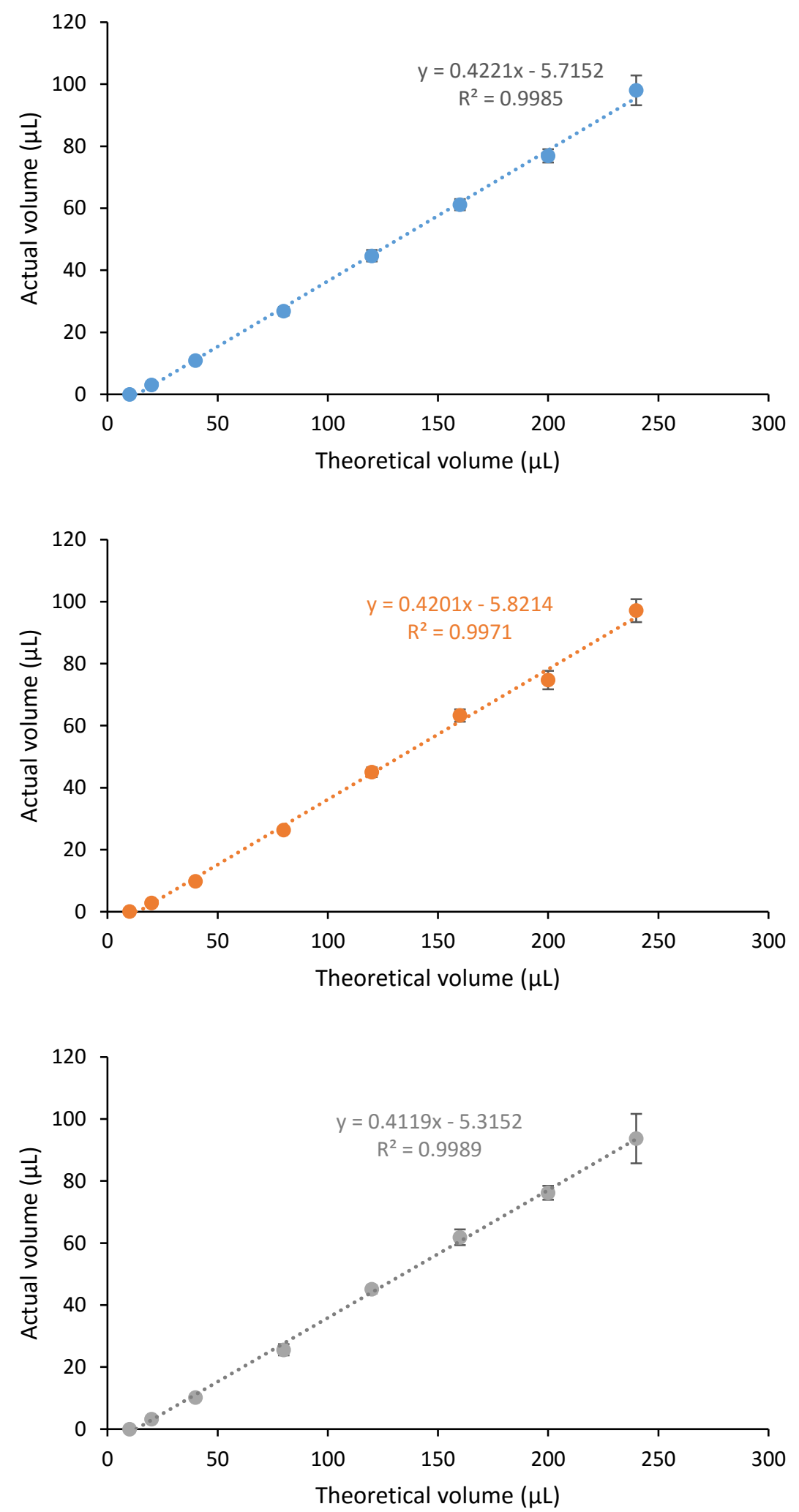

Figure S2 Linear relationship theoretical volume of the software and dispensed volume using singlephase printing mode and different nozzle aperture sizes (blue, orange and grey graphs for $0.25,0.41$ or $0.84 \mathrm{~mm}$ nozzles respectively). 

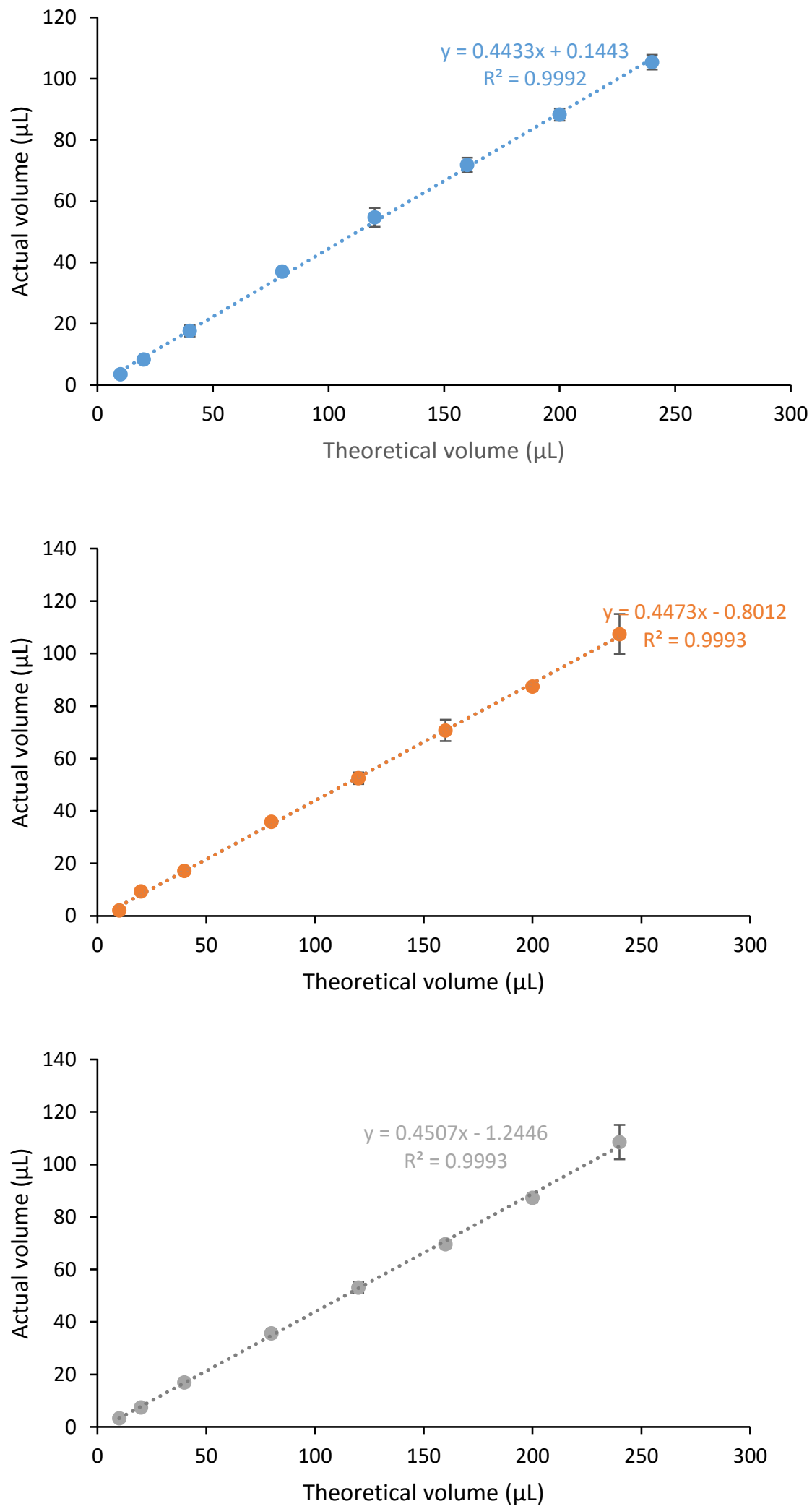

Figure S3 Linear relationship theoretical volume of the software and dispensed volume using multiphase printing mode and different nozzle aperture sizes (blue, orange and grey graphs for $0.25,0.41$ or $0.84 \mathrm{~mm}$ nozzles respectively). 